\title{
Maximum-likelihood dual-energy tomographic image reconstruction
}

\author{
Jeffrey A. Fessler ${ }^{a, b, c}$, Idris Elbakri ${ }^{a}$, Predrag Sukovic ${ }^{b}$, Neal H. Clinthorne ${ }^{c}$ \\ ${ }^{a}$ EECS Department, University of Michigan, Ann Arbor, MI, USA \\ ${ }^{b}$ BME Department, University of Michigan, Ann Arbor, MI, USA \\ ${ }^{c}$ Division of Nuclear Medicine, Dept. of Radiology, UM
}

\begin{abstract}
Dual-energy (DE) X-ray computed tomography (CT) has shown promise for material characterization and for providing quantitatively accurate CT values in a variety of applications. However, DE-CT has not been used routinely in medicine to date, primarily due to dose considerations. Most methods for DE-CT have used the filtered backprojection method for image reconstruction, leading to suboptimal noise/dose properties. This paper describes a statistical (maximum-likelihood) method for dual-energy X-ray CT that accommodates a wide variety of potential system configurations and measurement noise models. Regularized methods (such as penalized-likelihood or Bayesian estimation) are straightforward extensions. One version of the algorithm monotonically decreases the negative log-likelihood cost function each iteration. An ordered-subsets variation of the algorithm provides a fast and practical version.
\end{abstract}

Keywords: Statistical image reconstruction, dual-energy X-ray computed tomography, polyenergetic spectra

\section{INTRODUCTION}

Tomographic images of the spatial distribution of attenuation coefficients in the human body are valuable for medical diagnosis. Most hospitals have CT scanners for producing such images. Attenuation images are also useful in a variety of scientific studies, and in industry for nondestructive evaluation and for security purposes like baggage inspection. CT scanners are also being integrated into SPECT and PET scanners to provide accurate attenuation correction for emission image reconstruction and for precise anatomical localization of the functional features seen in the emission images.

The attenuation coefficients of all materials depend on the energy of the incident photons. In clinical X-ray CT imaging, the source of the $\mathrm{X}$-ray photons, bremsstrahlung radiation, has an inherently broad energy spectrum. Each photon energy is attenuated differently by the object (body). When such transmission measurements are processed by conventional image reconstruction methods, this energy-dependent effect causes beam-hardening artifacts and compromises quantitative accuracy. To avoid these difficulties, one could employ a radioisotope source with a monoenergetic spectrum, but the practical intensity is usually much lower leading to lower SNR. Higher intensities are obtained from monoenergetic synchrotron sources, which are expensive currently [1]. Many gamma-emitting radioisotopes also emit photons at several photon energies, and the methods described in this paper are also useful for systems that use gamma sources with multiple energies, such as some SPECT transmission scans [2].

We have previously developed a statistical method for reconstructing images from a single measured X-ray CT sinogram [3]. To our knowledge, that method was the first statistical approach to include a complete polyenergetic source spectrum model in a penalized-likelihood framework with a monotonically converging iterative algorithm. De Man $e t$ al. also proposed a solution to that problem based on a somewhat different object model and an algorithm that may not be monotonically converging [4]. When only a single sinogram (for a given polyenergetic source spectrum) is available, usually one must make some fairly strong assumptions about the object's attenuation properties to perform reconstruction. For example, one may segment the object into soft tissue and bone voxels [3,5-8] or mixtures thereof [9]. Using multiple measurements with "energy diversity," i.e., a set of two or more energy spectra, one can avoid this segmentation, eliminating one potential source of errors.

The energy dependence of attenuation coefficients is an inconvenience in conventional X-ray CT. Viewed from a broader perspective however, this dependence can be considered an asset in that it allows for the possibility of material characterization from transmission tomographic measurements. Early work by Alvarez and Macovski [10-13] showed

Email: fessler@umich.edu, Voice: 734-763-1434, Web: http://www.eecs . umich.edu/ fessler 
how to approximate the energy dependence of attenuation coefficients in terms of a Compton scattering component and a photoelectric absorption component (or, roughly equivalently, electron density and atomic number [14]) and how to separate these two components in the projection domain prior to tomographic reconstruction. The separate component images could then be combined to synthesize a displayed CT image at any energy of interest [15]. Later enhancements included noise suppression [16], considerations in basis material choices [17-22], energy optimization [23], beam-hardening assessment and correction [24,25], algorithm acceleration [26, 27], scatter correction [28], and evaluation of precision [29].

Numerous potential applications of dual-energy imaging have been explored, including rock characterization for petrochemical industrial applications [30,31], soil sample analysis in agriculture [32], bone mineral density measurements [33-39], bone marrow composition [40], adipose tissue volume determinations [41], liver iron concentrations [42, 43], explosives detection [44], detection of contrast agents in spinal canal [45], nondestructive evaluation [46], body composition [47], carotid artery plaques [1], and radioactive waste drums [48]. Accurate correction of Compton scatter in X-ray CT may also benefit from dual-energy information.

More recently, there has been considerable interest in using X-ray CT images to correct for attenuation in SPECT $[49,50]$ and PET image reconstruction [51]. In these contexts, one must scale the attenuation values in the X-ray CT images from the $\mathrm{X}$-ray photon energies to the energies of the gamma photons used in SPECT and PET imaging. Kinahan et al. have noted that accurate scaling from X-ray to PET energies may require dual-energy X-ray CT scans [51]. This is particularly challenging in the "arms down" mode of PET scanning. If the primary purpose of the dual-energy X-ray CT scan is PET attenuation correction (rather than diagnosis), then one would like to use low X-ray doses, resulting in the need for statistical image reconstruction methods to minimize image noise.

The conventional disadvantage of dual-energy methods is the increased scan time if two (or more) separate scans are acquired for each slice. This doubling in scan time can be avoided by methods such as alternating the source energy spectra between each projection angle [52] or between each slice [53] or conceivably in other arrangements. Special split detectors have also been proposed [54]. The method described in this paper is based on models that are sufficiently general to accommodate any such forms of energy diversity.

Prior to the 1990's, all work on dual-energy X-ray CT used the FBP reconstruction method. In the early 1990's there were a few iterative methods published for dual-energy CT reconstruction. Michael et al. presented an iterative method to achieve beam-hardening correction and decomposition into basis materials [55]. Markham and Fryar applied the ART algorithm [56]. Kotzki et al. applied a conjugate gradient algorithm [38]. These iterative approaches treat the problem as "finding the solution to a system of equations." These algebraic approaches can improve the accuracy relative to FBP methods, but they do not directly address the radiation dose issue. In contrast, in statistical image reconstruction approaches, the problem is posed as finding the images that best fit the measurements according to the (possibly nonlinear) physical model and a statistical model. Proper statistical modeling can lead to lower noise images, thereby enabling reductions in X-ray dose to the patient.

Statistical approaches have been extensively investigated, particularly in the last ten years, for monoenergetic transmission measurements; see [57] for a recent review. The method described in this paper is a novel extension of statistical image reconstruction approaches from the monoenergetic case to the case of measurements with energy diversity. We describe a statistical (maximum likelihood or penalized likelihood) method for reconstructing an "attenuation map" $\mu(\vec{x}, \mathcal{E})$ from polyenergetic X-ray (or gamma-ray) tomographic measurements.

Recently, Clinthorne and Sukovic have investigated iterative algorithms for dual-energy and triple-energy CT reconstruction based on a weighted least-squares approach, including object-domain constraints [58-62]. That work assumed monoenergetic measurements, whereas the proposed method uses a measurement model that accommodates a broad energy spectrum. Gleason et al. [63] also hint at the need for ML solutions to the multi-energy problem.

Like most dual-energy reconstruction methods, the proposed method requires some knowledge about the X-ray beam spectrum [64]. This spectrum can be measured directly [65] or estimated from calibration phantoms [66, 67]. In the final analysis, rather than requiring the entire spectrum, the algorithm requires only two nonlinear functions that may be feasible to measure empirically for a given scanner. We conjecture that the method will not exhibit inordinate sensitivity to imperfections in the source spectrum model. We plan to perform sensitivity analyses using tools such as those in [68].

Section 2 presents the physical model. Section 3 presents the statistical model. The derivation of the algorithm and reconstruction results will be submitted elsewhere, well before these proceedings finally arrive. In the first author's view, these proceedings are unreasonably inaccessible. Visit the first author's homepage for preprints and reprints. Section 4 discusses future directions. 


\section{PHYSICS AND OBJECT MODELS}

Let $\mu(\vec{x}, \mathcal{E})$ denote the object's linear attenuation coefficient as a function of spatial position $\vec{x}$ and photon energy $\mathcal{E}$. The ideal tomographic imaging system would provide a complete description of $\mu$ for $\vec{x}$ in the entire field of view and for a wide range of energies $\mathcal{E}$. In practice, the goal is to reconstruct an estimate of $\mu$ from a finite collection of "line-integral" measurements. (For simplicity we assume the object is static, and ignore any temporal variations, although it may be possible to generalize the results to the dynamic case [69].)

\subsection{General measurement physical model}

We assume the following general physical model for the measurements. We collect transmission tomographic measurements with $N_{s} \geq 1$ different incident spectra, e.g., by changing the X-ray source voltage and/or the source filtration. For each incident spectra, we record tomographic "line integrals" at $N_{d}$ radius-angle pairs, i.e., we form a sinogram (not necessarily completely sampled). Let $Y_{m i}$ denote the measurement for the $i$ th ray for the $m$ th incident spectrum, $m=1, \ldots, N_{s}, i=1, \ldots, N_{d}$. For notational simplicity we present the case where the same number of rays are recorded for each incident spectrum. The method generalizes easily to the case where the number or configuration of rays is different for different incident spectra, which may be useful in practice. We refer to $\left\{Y_{m i}\right\}_{i=1}^{N_{d}}$ as the measurements for the " $m$ th incident spectrum."

We assume that the measurements are random variables with the following ensemble means:

$$
E_{\mu}\left[Y_{m i}\right]=\bar{y}_{m i}[\mu] \triangleq \int I_{m i}(\mathcal{E}) \exp \left(-\int_{\mathcal{L}_{m i}} \mu(\vec{x}, \mathcal{E}) d \ell\right) d \mathcal{E}+r_{m i}
$$

where $\int_{\mathcal{L}_{m i}} \cdot d \ell$ denotes the "line integral" function for the $i$ th position and $m$ th energy ${ }^{*}$, and $I_{m i}(\mathcal{E})$ denotes the product of the source energy spectrum and the detector gain (for the $m$ th incident spectrum), and $r_{m i}$ denotes "known" additive background contibutions such as room background, dark current, and/or scatter (see discussion). We treat each $I_{m i}(\mathcal{E})$ and $r_{m i}$ as known and nonnegative. Determining $I_{m i}(\mathcal{E})$ in practice may require careful calibration procedures [66]. One usually determines $r_{m i}$ by some preprocessing steps prior to iterative reconstruction. For example, the $r_{m i}$ 's may be equated to known constants related to the "shifted Poisson" approach based on detector noise models [70-73].

This paper describes methods for reconstructing $\mu$ from tomographic measurements with energy diversity under loglikelihood models based on the general physical model (1). All previously published approaches have been based on simplifications of (1) or of the associated log-likelihoods, except [80]. We first describe those "conventional" simplifications, and then proceed to describe the new approach.

\subsection{Basis material decomposition (object model)}

We have only a finite set of measurements whereas $\mu$ is a continuous function of energy and spatial location. Parametric statistical estimation requires some form of discretization of $\mu$. For the polyenergetic case, one must parameterize both the spatial and energy dependencies. To our knowledge, all prior work has considered parameterizations that are separable in the spatial and energy (or material density) dimensions. Separable approaches seem simple and natural. For example, Alvarez and Macovski [10] assume that

$$
\mu(\vec{x}, \mathcal{E})=\sum_{l=1}^{N_{\mathrm{e}}} f_{l}(\mathcal{E}) \alpha_{l}(\vec{x})
$$

where each $f_{l}(\mathcal{E})$ depends only on energy but not on spatial position, $\alpha_{l}(\vec{x})$ is the corresponding coefficient that varies spatially, and $N_{\mathrm{e}}$ is usually 2 . Alternatively, Clinthorne et al. $[58,60,62]$ assume that

$$
\mu(\vec{x}, \mathcal{E})=\sum_{l=1}^{N_{\mathrm{e}}} \beta_{l}(\mathcal{E}) \rho_{l}(\vec{x}),
$$

where $\beta_{l}(\mathcal{E})$ denotes the energy-dependent mass-attenuation coefficient of the $l$ th material type (e.g., soft tissue, bone mineral, contrast agent, etc.), and $\rho_{l}(\vec{x})$ is the density of that material at spatial location $\vec{x}$. This latter parameterization

${ }^{*}$ Typically $\mathcal{L}_{m i}$ will be independent of $m$, except in systems such as [52] where alternate projection views have different energy spectra. 
facilitates enforcing physical constraints such as nonnegativity [58]. Both of the above parameterizations are separable in space / energy. This separability property is needed for the type of algorithm derived in [10] and [58,60,62]. The more general algorithm derived in this paper does not require separability. We describe a more general parameterization in (9) below after reviewing conventional approaches.

\subsection{Conventional dual-energy approach}

Substituting (2) into (1) yields the following simplified model for the measurement means:

$$
\bar{y}_{m i}=\int I_{m i}(\mathcal{E}) \exp \left(-\sum_{l} f_{l}(\mathcal{E}) \int_{\mathcal{L}_{m i}} \alpha_{l}(\vec{x}) d \ell\right) d \mathcal{E}+r_{m i}, \quad m=1, \ldots, N_{s} .
$$

Ignoring measurement noise, in the usual case where $\mathcal{L}_{m i}=\mathcal{L}_{i}$ is independent of $m$, one can view this expression for the $i$ th ray as a system of $N_{s}$ nonlinear equations in $N_{\mathrm{e}}$ unknowns, where the $l$ th unknown is $t_{l i} \triangleq \int_{\mathcal{L}_{i}} \alpha_{l}(\vec{x}) d \ell$, which is the $i$ th line integral through the $l$ th basis material. If $N_{s} \geq N_{\mathrm{e}}$, then for each $i$, one can solve these nonlinear equations by iterative methods or by polynomial approximation [10] yielding estimates $\hat{t}_{l i}$ of the $t_{l i}$ 's. Then one can apply either the conventional filtered back-projection (FBP) or a "conventional" iterative reconstruction algorithm separately to each sinogram $\left\{\hat{t}_{l i}\right\}_{i=1}^{N_{d}}$ to estimate the component images $\alpha_{l}(\vec{x})$. The FBP method usually yields unacceptably noisy estimates of the component images, hampering its acceptance. (Convex combinations of the component images have at best the same SNR as conventional X-ray CT images [15].) One could apply an iterative reconstruction method instead of FBP to estimate $\alpha_{l}(\vec{x})$ from the $\hat{t}_{l i}$ 's. For example, one could use error propagation methods, e.g., [68], to estimate the covariances of the $\hat{t}_{l i}$ 's and then estimate the $\alpha_{l}$ 's using a weighted least-squares cost function based on those covariances, e.g., [74]. Such an approach would be suboptimal statistically since the nonlinear processing that leads to the $\hat{t}_{l i}$ 's obscures their statistical distribution and seems to limit one to least-squares formulations. (Nevertheless, such approaches may be only "slightly" suboptimal at moderate dose levels so warrant further consideration.) Instead, we pursue a "preprocessing free" maximum-likelihood approach here.

\subsection{Conventional monoenergetic approximation}

Another way to simplify (1) is to assume that each incident spectrum is monoenergetic. That model is realistic for some radioisotope sources, but is a considerable idealization of X-ray sources. Mathematically, the monoenergetic assumption is expressed

$$
I_{m i}(\mathcal{E})=I_{m i} \delta\left(\mathcal{E}-\mathcal{E}_{m}\right),
$$

where $\mathcal{E}_{m}$ denotes the energy of the $m$ th setting, $m=1, \ldots, N_{s}$. Under this assumption, the model (1) simplifies to

$$
\bar{y}_{m i}=I_{m i} \exp \left(-\int_{\mathcal{L}_{m i}} \mu\left(\vec{x}, \mathcal{E}_{m}\right) d \ell\right)+r_{m i}
$$

In this case one can estimate the line integrals $\ell_{m i} \triangleq \int_{\mathcal{L}_{m i}} \mu\left(\vec{x}, \mathcal{E}_{m}\right) d \ell$ by a simple logarithm:

$$
\hat{\ell}_{m i} \triangleq \log \left(\frac{I_{m i}}{Y_{m i}-r_{m i}}\right) \approx \int_{\mathcal{L}_{m i}} \mu\left(\vec{x}, \mathcal{E}_{m}\right) d \ell
$$

Again, one could apply the FBP method to reconstruct $\mu\left(\vec{x}, \mathcal{E}_{m}\right)$ from $\left\{\hat{\ell}_{m i}\right\}_{i=1}^{N_{d}}$.

Clinthorne and Sukovic combined (6) with (3) to formulate a penalized weighted least-squares image reconstruction method for dual-energy and triple-energy tomographic reconstruction [58-62]. Their simulations matched the monoenergetic model (4), so the question of whether a monoenergetic approximation is adequate for iterative dual-energy tomographic image reconstruction is an open one. The algorithm proposed in this paper will facilitate comparisons between the full polyenergetic treatment and the simpler monoenergetic approximation.

The case of a single monoenergetic measurement, i.e., $N_{s}=1$ in (4), is the most extensively studied tomographic reconstruction problem, and numerous non-statistical and statistical methods have been proposed for this case. 
To estimate $\mu$ by iterative statistical methods, we must eventually parameterize it. In the single monoenergetic case, we usually assume

$$
\mu\left(\vec{x}, \mathcal{E}_{1}\right)=\sum_{j=1}^{N_{p}} b_{j}(\vec{x}) \mu_{j}
$$

for some spatial basis functions $b_{j}(\cdot)$, such as indicator functions over each pixel's support. Substituting into (5) yields

$$
\bar{y}_{1 i}[\mu]=I_{1 i} \exp \left(-\sum_{j=1}^{N_{p}} a_{i j} \mu_{j}\right)+r_{1 i},
$$

where

$$
a_{i j} \triangleq \int_{\mathcal{L}_{i}} b_{j}(\vec{x}) d \ell
$$

The model (7) is used in "conventional" statistical methods for transmission image reconstruction, e.g., [57, 75-77].

\subsection{Beam-hardening correction}

Elbakri and Fessler combined (3) with the polyenergetic measurement model (1) in the single scan case $\left(N_{s}=1\right)$ to develop a statistical method for X-ray CT image reconstruction with compensation for beam-hardening, assuming that the image can be segmented into soft-tissue and bone voxels [3]. This same assumption is used in conventional non-statistical methods for beam-hardening correction [5, 7,8]. De Man et al. proposed another statistical method for beam-hardening correction, assuming that all materials in the patient have spectral properties that are linear combinations of two basis materials [4]. An advantage of energy diversity approaches $\left(N_{s}>1\right)$ is that they eliminate the need for segmentation and other approximations that may hinder material characterization.

\subsection{Proposed polyenergetic approach}

As noted above, most prior work has considered object parameterizations that are separable in the spatial and energy (or material density) dimensions, as in (2) and (3). In the interest of generality here, we derive our algorithm under the following very flexible parameterization:

$$
\mu(\vec{x}, \mathcal{E})=\sum_{k=1}^{K_{b}} \chi_{k}(\vec{x}, \mathcal{E}) x_{k}
$$

where $K_{b}$ is the number of basis functions and $x_{k}$ is the unknown coefficient of the $k$ th basis function. By taking $K_{b}$ sufficiently large and using suitably localized $\chi_{k}$ 's, any function $\mu$ can be approximated to arbitrary accuracy by (9). Both of the preceding parameterizations (2) and (3) are special cases of (9). For the usual two-material separable parameterization, we have $K_{b}=2 N_{p}$ where $N_{p}$ is the number of voxels. A nonseparable basis may be useful for example if a certain material component (such as a metal implant) is known a priori to be present only in certain image locations. This may be useful even for the bone-mineral component if a priori segmentation can adequately identify the bone regions.

Using the general parameterization (9), the inner integral in (1) becomes:

$$
\int_{\mathcal{L}_{m i}} \mu(\vec{x}, \mathcal{E}) d \ell=\int_{\mathcal{L}_{m i}}\left[\sum_{k=1}^{K_{b}} \chi_{k}(\vec{x}, \mathcal{E}) x_{k}\right] d \ell=\sum_{k=1}^{K_{b}} a_{m i k}(\mathcal{E}) x_{k} \triangleq\left[\boldsymbol{A}_{m}(\mathcal{E}) \boldsymbol{x}\right]_{i}
$$

where the coefficient vector is $\boldsymbol{x} \triangleq\left(x_{1}, \ldots, x_{K_{b}}\right)$, and where $\boldsymbol{A}_{m}(\mathcal{E})$ is a $N_{d} \times K_{b}$ matrix with elements

$$
\left[\boldsymbol{A}_{m}(\mathcal{E})\right]_{i k}=a_{m i k}(\mathcal{E}) \triangleq \int_{\mathcal{L}_{m i}} \chi_{k}(\vec{x}, \mathcal{E}) d \ell
$$

for $i=1, \ldots, N_{d}, k=1, \ldots, K_{b}$. Substituting into (1) yields the following discrete-object discrete-data mean model:

$$
\bar{y}_{m i}(\boldsymbol{x})=\int I_{m i}(\mathcal{E}) e^{-\left[\boldsymbol{A}_{m}(\mathcal{E}) \boldsymbol{x}\right]_{i}} d \mathcal{E}+r_{m i} .
$$

In the absence of noise, our goal would be to estimate $\boldsymbol{x}$ from the measurements $\left\{Y_{m i}\right\}$ using the model (10). 


\section{STATISTICAL MODEL AND LIKELIHOOD}

This section describes our assumptions about the measurement statistics and formulates the log-likelihood.

\subsection{Statistical models}

If one used photon-counting detectors with modest deadtimes, then it would be reasonable to assume that the measurements are statistically independent Poisson random variables with means (1), i.e.,

$$
Y_{m i} \sim \operatorname{Poisson}\left\{\bar{y}_{m i}[\mu]\right\} .
$$

In this case, for a given measurement realization $Y_{m i}=y_{m i}$, the corresponding negative log-likelihood of $\boldsymbol{x}$ has the form

$$
-L(\boldsymbol{x}) \equiv \sum_{m=1}^{N_{s}} \sum_{i=1}^{N_{d}} \bar{y}_{m i}(\boldsymbol{x})-y_{m i} \log \bar{y}_{m i}(\boldsymbol{x}),
$$

where $\equiv$ means "equal to within irrelevant constants independent of $\boldsymbol{x}$." This is the model used in most statistical image reconstruction methods for transmission tomography to date [57] and it is natural for photon-counting detectors such as those used in PET and SPECT transmission scans.

Although photon-counting X-ray detectors do exist [78], commercial X-ray CT systems use current integrating detectors that yield energy-dependent signals and additional electronic noise variance beyond that due to Poisson counting variability [79]. To first order, additive electronic noise can be approximated within the Poisson model using the $r_{m i}$ terms in (1) by a simple modification of the "shifted Poisson" approach [70-73]. It is likely that the "exact" likelihood for such detectors is analytically intractable, so approximations will undoubtably be used in practice. For example, Clinthorne describes a sophisticated point-process model for X-ray detection and uses its first and second moments [80]. Rather than postulating and attempting to validate any particular approximate statistical model in this paper, we derive the algorithms under very general assumptions that will accommodate a wide variety of log-likelihood models and approximations that might be proposed in the future.

We make the following four assumptions about the measurement statistics.

1. The measurements $\left\{Y_{m i}\right\}$ are statistically independent.

Due to effects like scintillator afterglow and electronics lag, statistical independence may not hold exactly in practice, but it is likely to be an accurate approximation for most X-ray CT systems. Accounting for whatever statistical dependencies may be present in real systems would likely be quite challenging.

2. The marginal negative log-likelihood of $Y_{m i}$ has the form $\psi_{m i}\left(\bar{y}_{m i}(\boldsymbol{x})\right)$ for some scalar function $\psi_{m i}$. For example, if the measurements have Poisson distributions, then

$$
\psi_{m i}(y)=y-y_{m i} \log y .
$$

This is perhaps the simplest case, and the easiest one to keep in mind on the first readings, but we allow for much more general $\psi_{m i}$ 's in the derivation.

3. The final two assumptions are more technical and concern the existence of convenient surrogate functions for the $\psi_{m i}$ 's of interest. We believe that all physically plausible $\psi_{m i}$ 's will satisfy these quite general assumptions. They are certainly satisfied for Poisson and Gaussian statistical models, as shown in [75].

For each $\psi_{m i}$, we assume that there exists a corresponding scalar surrogate function $h_{m i}(\cdot, \cdot)$ that is convex on $(0, \infty)$ in its first argument. By surrogate function, we mean a function that satisfies

$$
\begin{array}{ll}
h_{m i}(y, y)=\psi_{m i}(y), & \forall y>0 \\
h_{m i}(y, z) \geq \psi_{m i}(z), & \forall y, z>0 .
\end{array}
$$

These conditions are the key to deriving an iterative algorithm that monotonically decreases the cost function defined below [75]. For each $z>0$, we also assume that $h_{m i}(\cdot, z)$ is differentiable in its first argument in an open interval around $z$. This assumption, combined with (12) and (13), ensures the following tangent condition:

$$
\dot{h}_{m i}(z, z)=\dot{\psi}_{m i}(z), \quad \forall z>0,
$$

where $\dot{h}_{m i}(y, z) \triangleq \frac{\partial}{\partial y} h(y, z)$. 
4. Convexity alone may be sufficient for some types of iterative minimization algorithms. However, to enable use of very simple descent methods we will follow the approach taken in most of our recent work by finding parabolic surrogates $[3,75,81]$. The following assumption ensures that the necessary parabola exists, which it certainly does in the Poisson case among others [75].

For any $\boldsymbol{x} \geq \mathbf{0}$, we assume that the following function

$$
g_{m i}(\ell, \boldsymbol{x}, \mathcal{E}) \triangleq h_{m i}\left(b_{m i}(\boldsymbol{x}, \mathcal{E}) e^{-\ell}+r_{m i}(\boldsymbol{x}, \mathcal{E}), \bar{y}_{m i}(\boldsymbol{x})\right)
$$

has a quadratic surrogate for $\ell \geq 0$, where we define the following functions for later use:

$$
\begin{aligned}
b_{m i}(\boldsymbol{x}, \mathcal{E}) & \triangleq \bar{y}_{m i}(\boldsymbol{x}) / t_{m i}(\boldsymbol{x}, \mathcal{E}) \\
t_{m i}(\boldsymbol{x}, \mathcal{E}) & \triangleq \exp \left(-\left[\boldsymbol{A}_{m}(\mathcal{E}) \boldsymbol{x}\right]_{i}\right)+r_{m i} / I_{m i} \\
I_{m i} & \triangleq \int I_{m i}(\mathcal{E}) d \mathcal{E} \\
r_{m i}(\boldsymbol{x}, \mathcal{E}) & \triangleq b_{m i}(\boldsymbol{x}, \mathcal{E}) r_{m i} / I_{m i} .
\end{aligned}
$$

In other words, we assume that there exists a curvature function $c_{m i}(\boldsymbol{x}, \mathcal{E})$ such that the following parabola is a surrogate for $g_{m i}$ :

$$
\begin{aligned}
q_{m i}(\ell, \boldsymbol{x}, \mathcal{E}) \triangleq \quad & g_{m i}\left(\left[\boldsymbol{A}_{m}(\mathcal{E}) \boldsymbol{x}\right]_{i}, \boldsymbol{x}, \mathcal{E}\right) \\
& +\dot{g}_{m i}\left(\left[\boldsymbol{A}_{m}(\mathcal{E}) \boldsymbol{x}\right]_{i}, \boldsymbol{x}, \mathcal{E}\right)\left(\ell-\left[\boldsymbol{A}_{m}(\mathcal{E}) \boldsymbol{x}\right]_{i}\right)+\frac{1}{2} c_{m i}(\boldsymbol{x}, \mathcal{E})\left(\ell-\left[\boldsymbol{A}_{m}(\mathcal{E}) \boldsymbol{x}\right]_{i}\right)^{2}
\end{aligned}
$$

where $\dot{g}_{m i}(\ell, \boldsymbol{x}, \mathcal{E}) \triangleq \frac{\partial}{\partial \ell} g_{m i}(\ell, \boldsymbol{x}, \mathcal{E})$. In assuming that $q_{m i}$ is a surrogate for $g_{m i}$, we mean that $c_{m i}$ is such that

$$
q_{m i}(\ell, \boldsymbol{x}, \mathcal{E}) \geq g_{m i}(\ell, \boldsymbol{x}, \mathcal{E}), \quad \forall \boldsymbol{x} \geq \mathbf{0}, \forall \ell \geq 0 .
$$

The construction (20) provides the following two surrogate properties:

$$
\begin{aligned}
\left.q_{m i}(\ell, \boldsymbol{x}, \mathcal{E})\right|_{\ell=\left[\boldsymbol{A}_{m}(\mathcal{E}) \boldsymbol{x}\right]_{i}} & =g_{m i}\left(\left[\boldsymbol{A}_{m}(\mathcal{E}) \boldsymbol{x}\right]_{i}, \boldsymbol{x}, \mathcal{E}\right) \\
\left.\dot{q}_{m i}(\ell, \boldsymbol{x}, \mathcal{E})\right|_{\ell=\left[\boldsymbol{A}_{m}(\mathcal{E}) \boldsymbol{x}\right]_{i}} & =\dot{g}_{m i}\left(\left[\boldsymbol{A}_{m}(\mathcal{E}) \boldsymbol{x}\right]_{i}, \boldsymbol{x}, \mathcal{E}\right) .
\end{aligned}
$$

\subsubsection{Existence of convex surrogates}

The existence of a differentiable convex surrogate $h_{m i}$ satisfying (12) and (13) always holds when $\psi_{m i}$ is twice differentiable, which it will always be for physically plausible statistical models.

Let $\psi(y)$ be any twice differentiable function and define

$$
h(y, z)=\psi(z)+\dot{\psi}(z)(y-z)+\int_{z}^{y}(y-\tau) \max \{\ddot{\psi}(\tau), \ddot{\psi}(a), 0\} d \tau .
$$

This surrogate $h$ is convex and (twice) differentiable and satisfies (12) and (13). The construction (22) may not be the optimal surrogate in terms of convergence rate, but it confirms that the third assumption above is unrestrictive.

The curvature of the surrogate $h(y, z)$ is $\max \{\ddot{\psi}(z), \ddot{\psi}(a), 0\}$. The " 0 " term ensures convexity of $h$, and the first two terms ensure that $h$ majorizes $\psi$, per (13).

Of course if $\psi_{m i}$ is itself convex, such as in the Poisson case, then we simply take $h_{m i}(y, \cdot)=\psi_{m i}(y)$.

\subsubsection{Existence of parabola surrogates}

To derive a specific algorithm for a particular negative $\log$-likelihood $\psi_{m i}$, one will need to determine the $c_{m i}$ function in (20) by careful analysis. In the case of Poisson measurements, where $\psi_{m i}(y)=h_{m i}(y, \cdot)=y-y_{m i} \log y$, the optimal $c_{m i}$ function was shown in $[57,75]$ to be

$$
c_{m i}^{\mathrm{opt}}(\boldsymbol{x}, \mathcal{E}) \triangleq c_{\mathrm{opt}}\left(\left[\boldsymbol{A}_{m}(\mathcal{E}) \boldsymbol{x}\right]_{i}, y_{m i}, b_{m i}(\boldsymbol{x}, \mathcal{E}), r_{m i}(\boldsymbol{x}, E)\right),
$$


where

$$
c_{\mathrm{opt}}(\ell, y, b, r)= \begin{cases}{\left[-2 \frac{z(\ell, y, b, r)}{\ell^{2}}\right]_{+},} & \ell>0 \\ {[-\ddot{g}(\ell, y, b, r)]_{+},} & \ell=0,\end{cases}
$$

where

$$
\begin{aligned}
z(\ell, y, b, r) & \triangleq g(0, y, b, r)-g(\ell, y, b, r)+\dot{g}(\ell, y, b, r) \ell \\
g(\ell, y, b, r) & =\left(b e^{-\ell}+r\right)-y \log \left(b e^{-\ell}+r\right) \\
\dot{g}(\ell, y, b, r) & =\frac{\partial}{\partial \ell} g=\left[1-\frac{y}{b e^{-\ell}+r}\right](-1) b e^{-\ell} \\
\ddot{g}(\ell, y, b, r) & =\frac{\partial^{2}}{\partial \ell^{2}} g=\left[1-\frac{y r}{\left(b e^{-\ell}+r\right)^{2}}\right] b e^{-\ell} .
\end{aligned}
$$

By "optimal," we mean the choice the leads to the fastest convergence [57,75].

It was also shown in [75, Theorem 1] that the curvature choice (24) is optimal not only for Poisson measurements, but also for a fairly broad family of negative log-likelihoods.

Alternatively, if $g_{m i}$ has bounded curvature, then one could use the upper bound on that curvature as the choice for $c_{m i}$. This approach was called "maximum curvature" in [75]. It is the simplest choice, but is suboptimal in terms of convergence rate. To summarize, assuming existence of parabola surrogates should not unduly restrict the class of statistical models.

\subsection{Likelihood formulation}

Under the above assumptions, including statistical independence of the transmission measurements, the negative loglikelihood corresponding to the above physical model has the form

$$
-L(\boldsymbol{x}) \equiv \Psi(\boldsymbol{x}) \triangleq \sum_{m=1}^{N_{s}} \sum_{i=1}^{N_{d}} \psi_{m i}\left(\bar{y}_{m i}(\boldsymbol{x})\right)
$$

for some scalar functions $\psi_{m i}$ that depend on the selected statistical model. Our goal is to estimate the coefficient vector $\boldsymbol{x}$ from the measurements $\left\{Y_{m i}\right\}$ by maximizing the log-likelihood or equivalently by finding a minimizer of the cost function $\Psi$ (or a regularized version thereof):

$$
\hat{\boldsymbol{x}}_{\mathrm{ML}} \triangleq \arg \min _{\boldsymbol{x}} \Psi(\boldsymbol{x}) .
$$

Optimization is restricted to the valid parameter space (i.e., including nonnegativity constraints etc.). Ignoring any constraints, in principle one could find a minimizer by zeroing the following partial derivatives of the cost function:

$$
\begin{aligned}
\frac{\partial}{\partial x_{k}} \Psi(\boldsymbol{x}) & =\sum_{m=1}^{N_{s}} \sum_{i=1}^{N_{d}} \dot{\psi}_{m i}\left(\bar{y}_{m i}(\boldsymbol{x})\right) \frac{\partial}{\partial x_{k}} \bar{y}_{m i}(\boldsymbol{x}) \\
& =\sum_{m=1}^{N_{s}} \sum_{i=1}^{N_{d}} \dot{\psi}_{m i}\left(\bar{y}_{m i}(\boldsymbol{x})\right) \cdot(-1) \int I_{m i}(\mathcal{E}) a_{m i k}(\mathcal{E}) e^{-\left[\boldsymbol{A}_{m}(\mathcal{E}) \boldsymbol{x}\right]_{i}} d \mathcal{E}
\end{aligned}
$$

where $\dot{\psi}_{m i}(y) \triangleq \frac{d}{d y} \psi_{m i}(y)$. In general there is no closed form solution to the set of $K_{b}$ equations (29), so iterative algorithms are required.

Although many algorithms have been proposed [57] for the monoenergetic problem (7), none of those previously proposed algorithms is suitable for minimizing the cost function $\Psi(\boldsymbol{x})$ in the polyenergetic case. The greatest difficulty is the integral over energy in (10). Substituting a summation for this integral does not significantly simplify the problem. Further difficulties arise due to the nonlinearity of Beer's law in (1), and due to the nonquadratic form of typical choices for $\psi_{m i}(c f .(11))$. Elsewhere we apply optimization transfer principles to derive an iterative algorithm that monotonically decreases the cost function each iteration. It should converge to a local minimizer, and should converge to the global minimizer if the cost function is unimodal. (The cost function is convex is in the monoenergetic case under the Poison model if the $r_{1 i}$ 's are zero.) Global convergence needs further examination. Many variations on this basic algorithm are possible, $c f$. [75]. 


\section{DISCUSSION}

This paper has described a statistical formulation for reconstructing dual-energy X-ray CT images. The method is applicable to related tomographic imaging problems having energy diversity. The method can accommodate a very wide variety of statistical models and is likely to be sufficiently general to cover all useful choices since the mathematical assumptions on $\psi_{m i}$ in Section 3.1 are quite flexible. Identifying suitable models remains an important problem.

For simplicity, we have used an approximate physical model that ignores the nonlinearity caused by the exponential edge-gradient effect $[82,83]$. Using optimization transfer methods similar to those used here, one could extend the algorithm derivation to account for this effect. Other blurring effects like detector after-glow, finite X-ray focal spot size, flying focal spot, detector response, could also be included.

The algorithm derivation itself is quite similar to that used for transmission tomography with overlapping beams [81]. Here the "overlap" is spectral rather than spatial.

Using a dual-energy approach eliminates the need for beam-hardening corrections, and the use of statistical methods should also reduce metal artifacts [84].

\section{ACKNOWLEDGMENTS}

This work was supported in part by NIH grants CA60711 and CA65637.

\section{REFERENCES}

1. F. A. Dilmanian et al., "Single- and dual-energy CT with monochromatic synchrotron X-rays," Phys. Med. Biol. 42, pp. 371-87, Feb. 1997.

2. A. Welch, G. T. Gullberg, P. E. Christian, L. Jia, and B. M. W. Tsui, "An investigation of dual energy transmission measurements in simultaneous transmission emission imaging," IEEE Tr. Nuc. Sci. 42, pp. 2331-8, Dec. 1995.

3. I. Elbakri and J. A. Fessler, "Ordered subsets transmission reconstruction with beam hardening correction for x-ray CT," in Proc. SPIE 4322, Medical Imaging 2001: Image Proc., 1, pp. 1-12, 2001.

4. B. De Man, J. Nuyts, P. Dupont, G. Marchal, and P. Suetens, "An iterative maximum-likelihood polychromatic algorithm for CT," IEEE Tr. Med. Im. 20, pp. 999-1008, Oct. 2001.

5. P. M. Joseph and R. D. Spital, "A method for correcting bone induced artifacts in computed tomography scanners," J. Comp. Assisted Tomo. 2, pp. 100-8, Jan. 1978.

6. O. Nalcioglu and R. Y. Lou, "Post-reconstruction method for beam hardening in computerised tomography," Phys. Med. Biol. 24(2), pp. 330-40, 1979.

7. P. M. Joseph and C. Ruth, "A method for simultaneous correction of spectrum hardening artifacts in CT images containing both bone and iodine," Med. Phys. 24, pp. 1629-34, Oct. 1997.

8. J. Hsieh, R. C. Molthen, C. A. Dawson, and R. H. Johnson, "An iterative approach to the beam hardening correction in cone beam CT," Med. Phys. 27, pp. 23-9, Jan. 2000.

9. C. H. Yan, R. T. Whalen, G. S. Beaupré, S. Y. Yen, and S. Napel, "Reconstruction algorithm for polychromatic CT imaging: Application to beam hardening correction," IEEE Tr. Med. Im. 19, pp. 1-11, Jan. 2000.

10. R. E. Alvarez and A. Macovski, "Energy-selective reconstructions in X-ray computed tomography," Phys. Med. Biol. 21(5), pp. 733-44, 1976.

11. A. Macovski, R. E. Alvarez, J. Chan, J. P. Stonestrom, and L. M. Zatz, "Energy dependent reconstruction in X-ray computerized tomography," Computers in Biology and Medicine 6, pp. 325-36, Oct. 1976.

12. R. E. Alvarez and A. Macovski, "X-ray spectral decomposition imaging system," 1977. U.S. Patent No. 4,029,963, June 14, 1977.

13. W. H. Marshall, R. E. Alvarez, and A. Macovski, "Initial results with prereconstruction dual-energy computed tomography (PREDECT)," Radiology 140, pp. 421-30, Aug. 1981.

14. M. R. Millner, W. D. McDavid, R. G. Waggener, M. J. Dennis, W. H. Payne, and V. J. Sauk, "Extraction of information from CT scans at different energies," Med. Phys. 6, pp. 70-1, Jan. 1979.

15. R. E. Alvarez and E. Seppi, "A comparison of noise and dose in conventional and energy selective computed tomography," IEEE Tr. Nuc. Sci. 26, pp. 2853-6, Apr. 1979.

16. W. A. Kalender, E. Klotz, and L. Kostaridou, "An algorithm for noise suppression in dual energy CT material density images," IEEE Tr. Med. Im. 7, pp. 218-24, Sept. 1988. 
17. J. B. Weaver and A. L. Huddleston, "Attenuation coefficients of body tissues using principal-components analysis," Med. Phys. 12, pp. 40-5, Jan. 1985.

18. D. J. Hawkes, D. F. Jackson, and R. P. Parker, "Tissue analysis by dual-energy computed tomography," British Journal of Radiology 59, pp. 537-402, June 1986.

19. J. C. M. Steenbeek, "Influence of calibration materials in single- and dual-energy quantitative CT," Radiology 183, pp. 849-55, June 1992.

20. J. C. M. Steenbeek, C. van Kuijk, J. L. Grashuis, and R. B. van Panthaleon van Eck, "Selection of fat-equivalent materials in postprocessing dual-energy quantitative CT," Med. Phys. 19, pp. 1051-6, July 1992.

21. K. L. Goh, S. C. Liew, and B. H. Hasegawa, "Correction of energy-dependent systematic errors in dual-energy X-ray CT using a basis material coefficients transformation method," IEEE Tr. Nuc. Sci. 44, pp. 2419-24, Dec. 1997.

22. K. L. Goh, S. C. Liew, and B. H. Hasegawa, "Energy-dependent systematic errors in dual-energy X-ray CT," IEEE Tr. Nuc. Sci. 44, pp. 212-7, Apr. 1997.

23. A. J. Talbert, R. A. Brooks, and D. G. Morgenthaler, "Optimum energies for dual-energy computed tomography," Phys. Med. Biol. 25, pp. 261-9, Mar. 1980.

24. A. J. Coleman and M. Sinclair, "A beam-hardening correction using dual-energy computed tomography," Phys. Med. Biol. 30, pp. 1251-6, Nov. 1985.

25. M. M. Goodsitt, "Beam hardening errors in post-processing dual energy quantitative computed tomography," Med. Phys. 22, pp. 1039-47, July 1995.

26. K.-S. Chuang and H. K. Huang, "A fast dual-energy computational method using isotransmission lines and table lookup," Med. Phys. 14, pp. 186-92, Mar. 1987.

27. H. N. Cardinal and A. Fenster, "An accurate method for direct dual-energy calibration and decomposition," Med. Phys. 17, pp. 327-41, May 1990.

28. J. R. Vetter and J. E. Holden, "Correction for scattered radiation and other background signals in dual-energy computed tomography material thickness measurements," Med. Phys. 15, pp. 726-31, Sept. 1988.

29. M. M. Goodsitt and R. H. Johnson, "Precision in quantitative CT: impact of X-ray dose and matrix size," Med. Phys. 19, pp. 1025-36, July 1992.

30. S. L. Wellington and H. J. Vinegar, "X-ray computerized tomography," J. Petroleum Technology 39(8), pp. 885-98, 1987.

31. C. C. Watson, "Optimal linear compression of x-ray transmission spectra," 1989. Schlumberger-Doll Research Note.

32. P. E. Cruvinel and F. A. Balogun, "Minitomography scanner for agriculture based on dual-energy Compton scattering," in SIBGRAPI 2000. 13th Brazilian Symposium on Computer Graphics and Image Processing. 17-20 Oct. 2000; Gramado, pp. 193-9, 2000.

33. P. B. Dunscombe, D. E. Katz, and A. J. Stacey, "Some practical aspects of dual-energy CT scanning," British Journal of Radiology 57, pp. 82-7, Jan. 1984.

34. J. R. Vetter, W. H. Perman, W. A. Kalender, R. B. Mazess, and J. E. Holden, "Evaluation of a prototype dual-energy computed tomographic apparatus. II. Determination of vertebral bone mineral content," Med. Phys. 13, pp. 340-3, May 1986.

35. A. E. Burgess, B. Colborne, and E. Zoffmann, "Vertebral trabecular bone: comparison of single and dual-energy CT measurements with chemical analysis," J. Comp. Assisted Tomo. 11, pp. 506-15, May 1987.

36. E. L. Nickoloff, F. Feldman, and J. V. Atherton, "Bone mineral assessment: new dual-energy CT approach," Radiology 168, pp. 223-8, July 1988.

37. M. A. Greenfield, "Current status of physical measurements of the skeleton," Med. Phys. 19, pp. 1349-57, Nov. 1992.

38. P. O. Kotzki, D. Mariano-Goulart, and M. Rossi, "Prototype of dual energy X-ray tomodensimeter for lumbar spine bone mineral density measurements; choice of the reconstruction algorithm and first experimental results," Phys. Med. Biol. 37, pp. 2253-65, Dec. 1992.

39. M. S. Westmore and M. Sato, "Effect of fat content on single- and dual-energy CT measurements of bone mineral: determination using a new system of tissue-mimicking phantom materials," in Proc. SPIE 3977, Phys. of Medical Imaging, pp. 609-19, 2000. 
40. M. M. Goodsitt, P. Hoover, M. S. Veldee, and S. L. Hsueh, "The composition of bone marrow for a dual-energy quantitative computed tomography technique. a cadaver and computer simulation study," Invest. Radiol. 29(7), pp. 695704, 1994.

41. H. Kvist, L. Sjostrom, and U. Tylen, "Adipose tissue volume determinations in women by computed tomography: technical considerations," Int. J. Obesity 10, pp. 53-67, 1986.

42. S. Oelckers, "In situ measurement of iron overload in liver tissue by dual-energy methods," Phys. Med. Biol. 41, pp. 1149-65, July 1996.

43. R. G. Sephton, "The potential accuracy of dual-energy computed tomography for the determination of hepatic iron," British Journal of Radiology 59, pp. 351-3, Apr. 1986.

44. F. L. Roder and R. P. Kruger, "Explosives detection by dual-energy computed tomography (CT)," in Proc. SPIE 182, Imaging Applications for Automated Industrial Inspection and Assembly, pp. 171-8, 1979.

45. M. Takai and M. Kaneko, "Discrimination between thorotrast and iodine contrast medium by means of dual-energy CT scanning," Phys. Med. Biol. 29, pp. 959-67, Aug. 1984.

46. P. Engler and W. D. Friedman, "Review of dual-energy computed tomography techniques," Materials Evaluation 48, pp. 623-9, May 1990.

47. J. F. Sutcliffe, "A review of in vivo experimental methods to determine the composition of the human body," Phys. Med. Biol. 41, pp. 791-833, May 1996.

48. C. Robert-Coutant, V. Moulin, R. Sauze, P. Rizo, and J. M. Casagrande, "Estimation of the matrix attenuation in heterogeneous radioactive waste drums using dual-energy computed tomography," Nucl. Instr. Meth. Phys. Res. A. 422, pp. 949-56, Feb. 1999.

49. E. L. Gingold and B. H. Hasegawa, "Systematic bias in basis material decomposition applied to quantitative dualenergy x-ray imaging," Med. Phys. 19, pp. 25-33, Jan. 1992.

50. B. H. Hasegawa, T. F. Lang, J. K. Brown, E. L. Gingold, S. M. Reilly, S. C. Blankespoor, S. C. Liew, B. M. W. Tsui, and C. Ramanathan, "Object-specific attenuation correction of SPECT with correlated dual-energy X-ray CT," IEEE Tr. Nuc. Sci. 40, pp. 1242-52, Aug. 1993.

51. P. E. Kinahan, D. W. Townsend, T. Beyer, and D. Sashin, "Attenuation correction for a combined 3d PET/CT scannter," Med. Phys. 25, pp. 2046-53, Oct. 1998.

52. W. A. Kalender, W. H. perman, J. R. Vetter, and E. Klotz, "Evaluation of a prototype dual-energy computed tomographic apparatus. 1. Phantom studies," Med. Phys. 13, pp. 334-9, May 1986.

53. M. J. Guy, I. A. Castellano-Smith, M. A. Flower, G. D. Flux, R. J. Ott, and D. Visvikis, "DETECT-dual energy transmission estimation CT-for improved attenuation correction in SPECT and PET," IEEE Tr. Nuc. Sci. 45, pp. 1261-7, June 1998.

54. F. Kelcz, P. M. Joseph, and S. K. Hilal, "Noise considerations in dual energy CT scanning," Med. Phys. 6, pp. 418-25, Sept. 1979. Erratum: Medical Physics, 7(4):388, July 1980.

55. G. J. Michael, "Tissue analysis using dual energy CT," Australasian Physical \& Engineering Sciences in Medicine 15, pp. 25-37, Mar. 1992.

56. C. Markham and J. Fryar, "Element specific imaging in computerised tomography using a tube source of X-rays and a low energy-resolution detector system," Nucl. Instr. Meth. A324, pp. 383-8, Jan. 1993.

57. J. A. Fessler, "Statistical image reconstruction methods for transmission tomography," in Handbook of Medical Imaging, Volume 2. Medical Image Processing and Analysis, M. Sonka and J. M. Fitzpatrick, eds., pp. 1-70, SPIE, Bellingham, 2000.

58. N. H. Clinthorne, "A constrained dual-energy reconstruction method for material-selective transmission tomography," Nucl. Instr. Meth. Phys. Res. A. 352, pp. 347-8, Dec. 1994.

59. P. Sukovic and N. H. Clinthorne, "Data weighted vs. non-data weighted dual energy reconstructions for X-ray tomography," in Proc. IEEE Nuc. Sci. Symp. Med. Im. Conf., 3, pp. 1481-3, 1998.

60. P. Sukovic and N. H. Clinthorne, "Basis material decomposition using triple-energy X-ray computed tomography," in IEEE Instrumentation and Measurement Technology Conference, Venice, 3, pp. 1615-8, 1999.

61. P. Sukovic and N. H. Clinthorne, "Design of an experimental system for dual energy X-ray CT," in Proc. IEEE Nuc. Sci. Symp. Med. Im. Conf., 2, pp. 1021-2, 1999.

62. P. Sukovic and N. H. Clinthorne, "Penalized weighted least-squares image reconstruction in single and dual energy X-ray computed tomography," IEEE Tr. Med. Im. 19, pp. 1075-81, Nov. 2000. 
63. S. S. Gleason, H. Sari-Sarraf, M. J. Paulus, D. K. Johnson, S. J. Norton, and M. A. Abidi, "Reconstruction of multi-energy X-ray computed tomography images of laboratory mice," IEEE Tr. Nuc. Sci. 46, pp. 1081-6, Aug. 1999.

64. U. Hassler, L. Garnero, and P. Rizo, "X-ray dual-energy calibration based on estimated spectral properties of the experimental system,” IEEE Tr. Nuc. Sci. 45, pp. 1699-1712, June 1998.

65. K. Aoki and M. Koyama, "A silicon diode in a thimble-type mount for measurement of diagnostic X-ray spectra," Phys. Med. Biol. 35, pp. 1505-18, Nov. 1990.

66. C. Ruth and P. M. Joseph, "Estimation of a photon energy spectrum for a computed tomography scanner," Med. Phys. 24, pp. 695-702, May 1997.

67. C. H. Yan, R. T. Whalen, G. S. Beaupré, S. Y. Yen, and S. Napel, "Modeling of polychromatic attenuation using computed tomography reconstructed images," Med. Phys. 26, pp. 631-42, Apr. 1999.

68. J. A. Fessler, "Mean and variance of implicitly defined biased estimators (such as penalized maximum likelihood): Applications to tomography," IEEE Tr. Im. Proc. 5, pp. 493-506, Mar. 1996.

69. N. P. Willis and Y. Bresler, "Optimal scan for time-varying tomography i: Theoretical analysis and fundamental limitations,” IEEE Tr. Im. Proc. 4, pp. 642-53, May 1995.

70. M. Yavuz and J. A. Fessler, "Statistical image reconstruction methods for randoms-precorrected PET scans," Med. Im. Anal. 2(4), pp. 369-378, 1998.

71. M. Yavuz and J. A. Fessler, "Penalized-likelihood estimators and noise analysis for randoms-precorrected PET transmission scans," IEEE Tr. Med. Im. 18, pp. 665-74, Aug. 1999.

72. D. L. Snyder, A. M. Hammoud, and R. L. White, "Image recovery from data acquired with a charge-couple-device camera," J. Opt. Soc. Am. A 10, pp. 1014-23, May 1993.

73. D. L. Snyder, C. W. Helstrom, A. D. Lanterman, M. Faisal, and R. L. White, "Compensation for readout noise in CCD images," J. Opt. Soc. Am. A 12, pp. 272-83, Feb. 1995.

74. J. A. Fessler, "Penalized weighted least-squares image reconstruction for positron emission tomography," IEEE Tr. Med. Im. 13, pp. 290-300, June 1994.

75. H. Erdoğan and J. A. Fessler, "Monotonic algorithms for transmission tomography," IEEE Tr. Med. Im. 18, pp. 80114, Sept. 1999.

76. H. Erdoğan and J. A. Fessler, "Ordered subsets algorithms for transmission tomography," Phys. Med. Biol. 44, pp. 2835-51, Nov. 1999.

77. F. J. Beekman and C. Kamphuis, “Ordered subset reconstruction for X-ray CT,” Phys. Med. Biol. 46, pp. 1835-55, July 2001.

78. H. Kruger, M. Lindner, P. Fischer, M. Lacker, S. Krimmel, N. Wermes, M. Koudag, G. Sato, T. Takahashi, and S. Watanage, "A X-ray imaging system using a single photon counting cdTe detector," in Proc. IEEE Nuc. Sci. Symp. Med. Im. Conf., pp. MR-1, 2001.

79. B. R. Whiting, L. J. Montagnino, and D. G. Politte, "Modeling X-ray computed tomography sinograms," 2001. submitted to mp.

80. N. H. Clinthorne, "Are hydrogenated amorphous silicon arrays usable for tomographic imaging?," IEEE Tr. Nuc. Sci. 41, pp. 1516-21, Aug. 1994.

81. D. F. Yu, J. A. Fessler, and E. P. Ficaro, "Maximum likelihood transmission image reconstruction for overlapping transmission beams," IEEE Tr. Med. Im. 19, pp. 1094-1105, Nov. 2000.

82. P. M. Joseph and R. D. Spital, "The exponential edge-gradient effect in X-ray computed tomography," Phys. Med. Biol. 26(3), pp. 473-87, 1981.

83. B. De Man, J. Nuyts, P. Dupont, G. Marchal, and P. Suetens, "Metal streak artifacts in X-ray computed tomography: a simulation study," IEEE Tr. Nuc. Sci. 46, pp. 691-6, June 1999.

84. G. Wang, D. L. Snyder, J. A. O'Sullivan, and M. W. Vannier, "Iterative deblurring for CT metal artifact reduction," IEEE Tr. Med. Im. 15, p. 657, Oct. 1996. 